\title{
ON SOME STRUCTURAL AND SPECTROSCOPIC ASPECTS OF RARE EARTH OXYCOMPOUNDS*
}

\author{
J. HöLsä ${ }^{\dagger}$, E. SÄILYNoJA \\ University of Turku, Department of Chemistry, FIN-20014 Turku, Finland \\ AND P. PORCHER \\ C.N.R.S., U.P.R. 209, F-92195 Meudon, France
}

(Received June 24, 1996)

\begin{abstract}
The aim of this account is to present the use and the advantages of different experimental and theoretical methods in the study of the structural and spectroscopic properties of rare earth (RE) oxyfluorides. The structural characterization was carried out with the X-ray and neutron powder diffraction techniques combined with the analysis of the acquired data with the Rietveld profile refinement method. The detailed spectroscopic studies were also used by employing the optical absorption and luminescence as well as inelastic neutron scattering data. Simple spectroscopic measurements gave, however, only qualitative description of the spectroscopic properties studied. More sophisticated and quantitative means was obtained by the application of the phenomenological crystal field theory to the spectroscopic data. On the other hand, the structural data was also used as initial input to electrostatic point charge calculation in order to extract the spectroscopic information. The structural and spectroscopic studies comprised the verification of the exact crystal and energy level structures and the characterization of the different interactions modifying the spectroscopic properties of the $\mathrm{RE}^{3+}$ ions. Finally, the results from the theoretical model were used to explain the evolution of the crystal field effect on the $4 f^{N}$ energy level structure of the $\mathrm{RE}^{3+}$ ion in the RE oxyfluorides series.
\end{abstract}

PACS numbers: $61.10 .-\mathrm{i}, 61.12 .-\mathrm{q}, 61.50 .-\mathrm{f}, 61.66 . \mathrm{Fn}$

\section{Introduction}

The detailed analysis of the $4 f^{N}$ energy level schemes of the trivalent rare earth ions [1] is of primordial importance considering many practical applications, e.g. those based on the energy transfer processes between alike or different rare

*This paper has been presented at the 2nd Winter Workshop on Spectroscopy and Structure of Rare Earth Systems, Polanica Zdrój, Poland, 1996.

tCorresponding author. 
earth ions. This is in contrast to the $d$-transition metals where the large-band structure of the electronic spectra allow much more freedom for those processes to take place. The experimental investigation of the energy level schemes is mostly time consuming - if not impossible in certain wavelength regions. Accordingly, there exists a definite need to acquire the necessary data from some model calculations. As the true $a b$ initio calculations are of practically no use in determining the internal structure of the $4 f^{N}$ configurations, some more simple models should be looked for.

In this paper we present a comprehensive study of the $4 f^{N}$ energy level structure of the $\mathrm{RE}^{3+}$ ions in the RE oxyfluoride (REOF) matrices, starting on one hand from the determination of the crystal structure by the Rietveld method and then applying a simple electrostatic point charge model to extract the $4 f^{N}$ energy level schemes. On the other hand, an unambiguous description for the $4 f^{N}$ energy level structure of the $\mathrm{RE}^{3+}$ ions was obtained through the phenomenological parametrization of the raw data consisting of the experimental energy levels. Finally, a comparison between the results from these two models is presented and the theoretical model is used to explain the evolution of the crystal field (c.f.) on the $4 f^{N}$ energy level structure of the $\mathrm{RE}^{3+}$ ion in the REOF series.

\section{Experimental}

\subsection{Sample preparation}

The polycrystalline samples of RE oxyfluorides, REOF (RE = La, Nd, Sm, $\mathrm{Gd}, \mathrm{Dy}$, and $\mathrm{Y}$ ) were prepared with a solid state reaction between the RE oxides, $\mathrm{RE}_{2} \mathrm{O}_{3}$ (minimum purity 99.99 mole\%) and ammonium fluoride, $\mathrm{NH}_{4} \mathrm{~F}$ [2]. In order to obtain the stoichiometric hexagonal oxyfluorides reaction was carried out in the following conditions: the temperature was between 850 and $1050^{\circ} \mathrm{C}$ for one to $1.5 \mathrm{~h}$ and the $\mathrm{NH}_{4} \mathrm{~F} / \mathrm{RE}_{2} \mathrm{O}_{3}$ ratio was between 2.0 and 4.5. The routine $\mathrm{X}$-ray powder diffraction (XPD) analysis was used to verify the structure and purity of oxyfluorides but no additional phases were observed.

\subsection{Powder diffraction}

The powder diffraction data for the pure stoichiometric REOF samples were collected for one hour at room temperature with an Enraf-Nonius PDS120 X-ray powder diffractometer using a position sensitive INEL CPS120 detector [3]. The monochromatic $\mathrm{Cu} K_{\alpha_{1}}$ radiation $(\lambda=1.5406 \AA)$ was used at the $2 \theta$ region between 6.5 and $120^{\circ}$. The angular resolution of the equipment was better than $0.018^{\circ}$. The external calibration was carried out with a silicon powder $2 \theta / d$-spacing standard (NBS 640b). The neutron diffraction experiment on NdOF was carried out on a conventional multichannel D2B high resolution two-axis diffractometer at ILL (Grenoble, France) at ambient temperature [4].

\subsection{Spectroscopic measurements}

The luminescence of the $\mathrm{RE}^{3+}$ doped $\mathrm{LaOF}, \mathrm{GdOF}$, and YOF was excited either by UV radiation from a high-pressure mercury lamp or by the blue (457.9) 
or green $(488.0 \mathrm{~nm})$ lines of an $\mathrm{Ar}^{+}$ion laser. The detailed experimental setups have been described in detail elsewhere $[5,6]$.

The optical absorption spectra of the pure REOF samples were measured by a Cary $5 \mathrm{E}$ UV-Vis-NIR spectrometer at selected temperatures between 9 and $300 \mathrm{~K}$ from 170 to $3300 \mathrm{~nm}$. The instrument reproducibility was better than $2 \AA$ and the band width used was $0.6 \AA$. The samples were prepared by mixing REOF to $\mathrm{KBr}$ and pressing a transparent disk. The inelastic neutron scattering measurements were carried out at ILL (Grenoble, France) by using an IN4 time of flight spectrometer in the 10 to $100 \mathrm{meV}$ thermal neutron energy range [4].

\section{Model calculations}

\subsection{Rietveld profile refinement}

The XPD and neutron powder diffraction data were refined by the Rietveld analysis [7] to obtain the exact crystal structure of the RE oxyfluorides. In the Rietveld method, the entire diffraction pattern is simulated starting from at least partly known structural model. The positions of the Bragg reflections (unit cell parameters), their intensities (atomic positional and thermal parameters) and reflection profiles were all refined by the least squares method. The actual calculations were carried out by the program DBWS-9006PC [8].

The results of the Rietveld analysis yielded a good agreement between the measured and calculated diffraction patterns with the $R_{\mathrm{wp}}$ values down to 0.05 . For the neutron diffraction analysis of NdOF (Fig. 1), this value was slightly higher, 0.07 . The atomic positions to be used later as an input to the point charge model

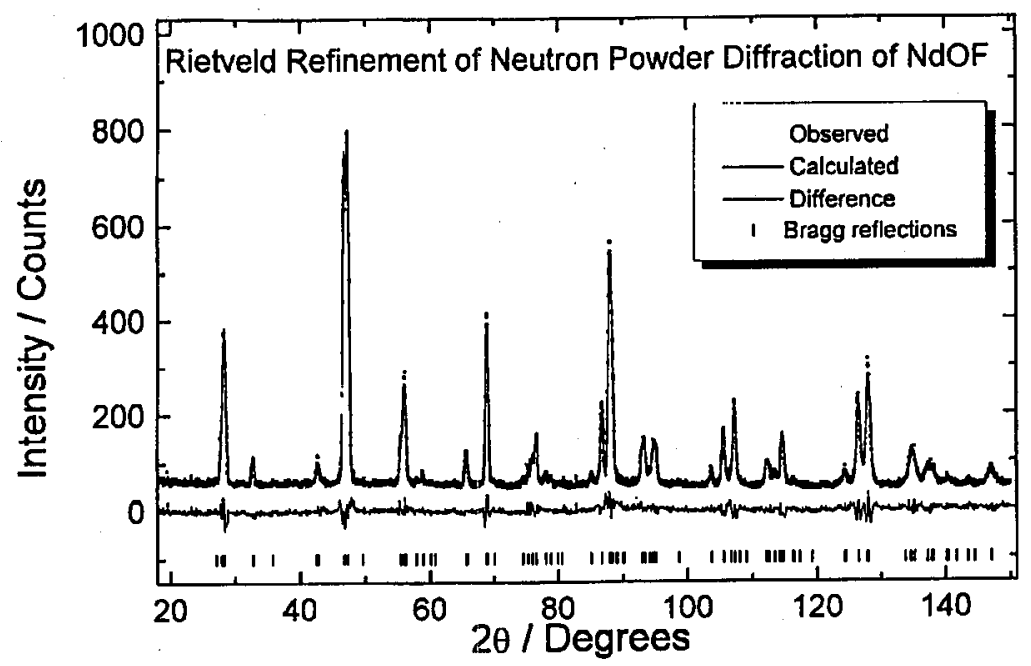

Fig. 1. Observed (scatter), calculated (solid line) and difference (lower) neutron powder diffraction patterns as well as the positions of the Bragg reflections for NdOF $(\lambda=$ $1.549 \AA)$. 
were determined with good accuracy. However, due to the presence of the heavy $\mathrm{RE}$ atom in the structure, the positions of the lighter oxygen and fluorine atoms were less well determined when the $\mathrm{X}$-ray diffraction was used.

\subsection{Phenomenological simulation of the energy level schemes}

The most important interactions describing the free ion energy level structure of the $\mathrm{RE}^{3+}$ ions are the Coulomb and spin-orbit interactions. The weaker interactions to be accounted for include the configuration interactions and the c.f. effect. The effective Hamiltonian $H$ can then be described as follows [9, 10]:

$$
\begin{aligned}
H= & H_{0}-\sum_{k=0,1,2,3} E_{k}(n f, n f) \mathrm{e}^{k}+\zeta_{4 f} A_{\mathrm{SO}}+\alpha L(L+1)+\beta G\left(G_{2}\right) \\
& +\gamma G\left(R_{7}\right)+\sum_{k=2,3,4,6,7,8} T^{k} t_{k}+\sum_{k, q, i} B_{q}^{k} C_{q}^{k}(i),
\end{aligned}
$$

where $E_{k}$ (Racah parameters) and $\zeta_{4 f}$ describe the electrostatic and spin-orbit interactions whereas $\alpha, \beta, \gamma$, and $T^{k}$ are associated with the two- and three-body configuration interactions, respectively. These are usually treated as parameters to be refined from experimental data. Moreover, for the $\mathrm{RE}^{3+}$ ions the free ion parameters vary only slightly with the matrix and can be even considered as constants for many practical applications.

The one-electron c.f. Hamiltonian is given by the sum of products between the c.f. parameters $B_{q}^{k}$ and the spherical tensors $C_{q}^{k}$. The number of the parameters is limited by the $C_{3 v}$ symmetry of the $\mathrm{RE}^{3+}$ site in REOF to six: $B_{0}^{2}, B_{0}^{4}, B_{3}^{4}, B_{0}^{6}, B_{3}^{6}$, and $B_{6}^{6}[11]$.

\subsection{Modified point charge model calculations}

The theoretical treatment of the electronic structure of the heavy atoms or ions as rare earths requires frequently drastic approximations. The simplest method used is the modified point charge model (PCEM) which accounts only for the point charge effects [12]. The inadequacies, i.e. the covalence in bonding, are partly compensated by the shielding $\sigma_{k}$ and expansion $\tau$ factors [13]. The $B_{q}^{k}$ parameters can then be expressed with the aid of the lattice sum parameters $A_{q}^{k}$ and the free ion radial integrals $\left\langle r^{k}\right\rangle$ :

$$
B_{q}^{k}=\tau^{-k}\left(1-\sigma_{k}\right) A_{q}^{k}\left\langle r^{k}\right\rangle \text {. }
$$

In addition to the crystal structure, the lattice sums depend strongly on the charge of the ions. In the present calculations, the effective charges +1.8 , -1.2 , and -0.6 , were used for the $\mathrm{RE}^{3+}$, oxide, and fluoride ions, respectively. The exact expression to calculate the $A_{q}^{k}$ lattice sum parameters has been presented elsewhere [6].

\section{Energy level simulations}

The experimental optical and inelastic scattering spectra of the $\mathrm{RE}^{3+}$ ions in REOF were analyzed in terms of the selection rules for the $C_{3 v}$ site symmetry. As a result, the experimental energy level schemes describe in details each electron 
configuration up to $c a .36000 \mathrm{~cm}^{-1}$ above which the dopant-host interactions hamper the experimental observations. For the $\mathrm{Fu}^{3+}$ and $\mathrm{Tb}^{3+}$ ions, however, only the ground $F$ septet was included in the simulations. Up to 195 c.f. levels $\left(\mathrm{Sm}^{3+}\right.$ in $\left.\mathrm{SmOF}\right)$ could be resolved from the experimental data, which means that even with a model including 14 free ions and six c.f. parameters accurate and physically meaningful simulations could be anticipated. The overall quality of the simulations was good with the rms deviations between the experimental and calculated energy level values usually around $17 \mathrm{~cm}^{-1}$; for $\mathrm{Eu}^{3+}$ and $\mathrm{Tb}^{3+}$ even less than $10 \mathrm{~cm}^{-1}$.

\subsection{Evolution of the free ion interactions}

The comparison of the free ion interactions across the $\mathrm{RE}^{3+}$ series is somewhat handicapped by the lack of sufficiently large data set. However, even now it is clear that the expected trend of an increase in the electrostatic repulsion between the $4 f$ coupling can be observed from $\mathrm{Pr}^{3+}$ to $\mathrm{Dy}^{3+}$ (Table I). The electrons and in the spin-orbit variation of the Slater integrals - calculated from the Racah parameters - follows rather well the evolution of the Dirac-Fock values [14]. The

TABLE I

The free ion parameter values $\left(\mathrm{cm}^{-1}\right)$ for YOF:Pr ${ }^{3+}, \mathrm{NdOF}, \mathrm{SmOF}$, and DyOF.

\begin{tabular}{c|c|c|c|c}
\hline \hline Parameter & YOF:Pr $^{3+}$ & NdOF & SmOF & DyOF \\
\hline$E_{0}$ & $9766(2)$ & $23429(1)$ & $46306(1)$ & $55250(1)$ \\
$E_{1}$ & $4507(2)$ & $4689(1)$ & $5190(0.2)$ & $6132(0.2)$ \\
$E_{2}$ & $21.14(2)$ & $23.17(1)$ & $25.45(1)$ & $30.40(1)$ \\
$E_{3}$ & $456.8(1)$ & $477.95(8)$ & $518.1(1)$ & $623.84(5)$ \\
$\alpha$ & $20.12(7)$ & $21.62(3)$ & $19.24(2)$ & $18.11(5)$ \\
$\beta$ & $-604(8)$ & $-625(3)$ & $-562(2)$ & $-603(2)$ \\
$\gamma$ & $1480(2)$ & $1880(4)$ & $1841(1)$ & $1600(1)$ \\
$T^{2}$ & & $424(2)$ & $306(1)$ & $350(1)$ \\
$T^{3}$ & & $52(2)$ & {$[33]^{a}$} & {$[78]^{a}$} \\
$T^{4}$ & & $55(2)$ & {$[94]^{a}$} & {$[41]^{a}$} \\
$T^{6}$ & & $-282(5)$ & $-248(8)$ & $-360(11)$ \\
$T^{7}$ & & $379(7)$ & $314(5)$ & $350(8)$ \\
$T^{8}$ & & $312(7)$ & $278(4)$ & $345(6)$ \\
$\zeta_{4 f}$ & $742(1)$ & $870(1)$ & $1147(1)$ & $1915(1)$ \\
\hline No. of levels & $58 / 91$ & $122 / 182^{b}$ & $195 / 1001^{b}$ & $153 / 1001^{b}$ \\
\hline$\sigma$ & 15 & 17 & 17 & 17 \\
\hline$\sigma$
\end{tabular}

${ }^{a}$ The $T^{3}$ and $T^{4}$ parameters in square brackets were not varied freely.

${ }^{b}$ Kramers doublets. 
deviations may be explained in most cáses by experimental uncertainty. Apart from the electrostatic repulsion and the spin-orbit coupling, the evolution of the other interactions, e.g. the configuration interaction, is not clear.

\subsection{Evolution of the crystal field effect}

In contrast to the free ion interactions, the data available on the c.f. parameters allows a detailed comparison of the c.f. effect across the REOF series up to DyOF. The existence of different host matrices involved in the data set may slightly hinder the comparison, which may also be the case with the difference in the size of the host and dopant cations. The evolution of the individual c.f. parameters in the REOF (Table II) is rather smooth. Following the simple reasoning of the point charge model, the c.f. effect should decrease with increasing atomic number due to the increasing nuclear charge experienced by the $4 f$ electrons. This seems to hold up to the middle of the series but beyond the $\mathrm{Gd}^{3+}$ ion, the c.f. effect seems, however, to regain some strength. Most clearly this behavior is illustrated by the evolution of the c.f. strength parameters (Fig. 2). Several explanations may be offered to resolve this anomaly. First, the one-electron c.f. operator alone may be inadequate to describe the c.f. interaction for the heavier rare earths. This could be taken into account by introducing the two-electron correlation effects $[17,18]$. However, their effect seems to be rather small and, moreover, be limited to certain free ion levels only [19].

The cancellation of the influence of the nuclear charge on the c.f. parameters can be deduced also from a more detailed treatment of the electrostatic point charge model where the $B_{q}^{k}$ parameters are given as products of the lattice sum parameters $A_{q}^{k}$ and the radial integrals $\left\langle r^{k}\right\rangle$ (Eq. (2)). For the latter half of the

TABLE II

The c.f. parameter values $\left(\mathrm{cm}^{-1}\right)$ for YOF: $\mathrm{Pr}^{3+}, \mathrm{NdOF}, \mathrm{SmOF}, \mathrm{GdOF}: \mathrm{Eu}^{3+}$, GdOF:Tb ${ }^{3+}$, and DyOF. For the definition of the c.f. strength parameters $S$ and $S^{k}$ $(k=4$ and 6$)$, see [15] and [16].

\begin{tabular}{c|c|c|c|c|c|c}
\hline \hline Parameter & YOF:Pr & NdOF & SmOF & GdOF:Eu & GdOF:Tb & DyOF \\
\hline$B_{0}^{2+}$ & $-124(16)$ & $-144(15)$ & $-200(22)$ & $-3(32)$ & $-27(25)$ & $57(16)$ \\
$B_{0}^{4}$ & $1612(32)$ & $1839(27)$ & $1786(29)$ & $1230(49)$ & $1522(37)$ & $1717(31)$ \\
$B_{3}^{4}$ & $-2276(16)$ & $-1643(17)$ & $-1308(21)$ & $-1588(24)$ & $-1494(21)$ & $-1272(21)$ \\
$B_{0}^{6}$ & $1237(33)$ & $1005(31)$ & $1139(35)$ & $1029(45)$ & $1059(32)$ & $1204(35)$ \\
$B_{3}^{6}$ & $1096(31)$ & $866(21)$ & $739(31)$ & $431(38)$ & $527(31)$ & $556(31)$ \\
$B_{6}^{6}$ & $745(32)$ & $812(22)$ & $810(31)$ & $786(34)$ & $490(26)$ & $640(31)$ \\
\hline$S$ & 780 & 650 & 580 & 556 & 554 & 543 \\
$S^{4}$ & 2379 & 2324 & 2405 & 1905 & 2135 & 2418 \\
$S^{6}$ & 2245 & 1957 & 1924 & 1633 & 1469 & 1699 \\
\hline No. of levels & $58 / 91$ & $122 / 182^{a}$ & $195 / 1001^{a}$ & $21 / 49^{b}$ & $19 / 49^{b}$ & $153 / 1001^{a}$ \\
\hline$\sigma$ & 15 & 17 & 17 & 11 & 6 & 17 \\
\hline
\end{tabular}

${ }^{a}$ Kramers doublets.

${ }^{b}$ Only the ${ }^{7} F$ term was used for the energy level simulation. 


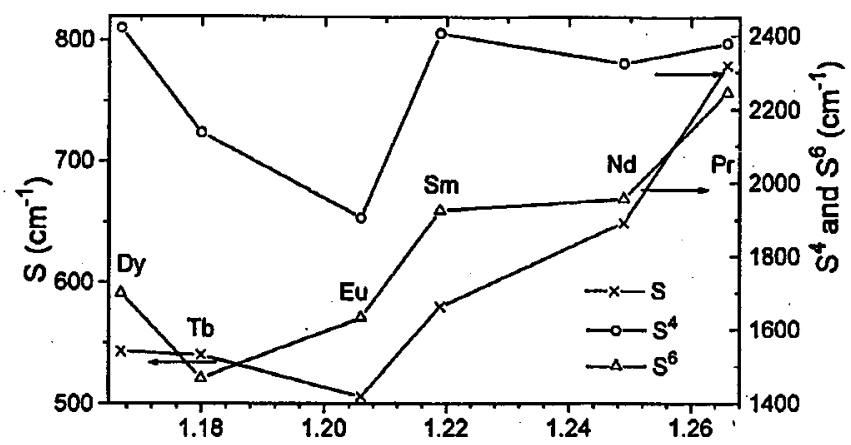

Fig. 2 lonic radius $(A)$
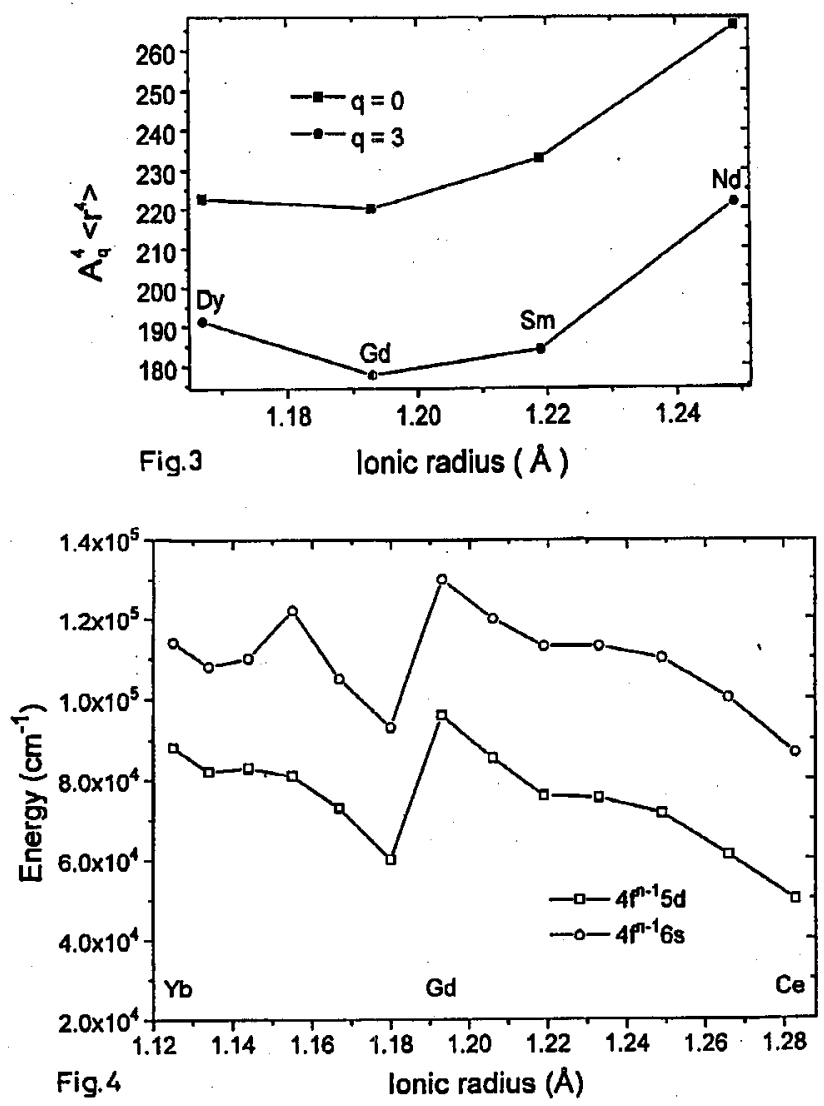

Fig. 2. Evolution of the c.f. strength parameters in the REOF series. For definition of the parameters, see [15] and [16].

Fig. 3 Evolution of the product between the lattice sum parameters $A_{q}^{k}$ and the free ion radial integrals $\left\langle r^{k}\right\rangle$ in the REOF series.

Fig. 4 Energies of some excited electron configurations in the $R E^{3+}$ series [20]. 
$\mathrm{RE}^{3+}$ series, the decrease in the radial integrals is smoothed out while the lattice sums keep on increasing in the REOF matrices. The combined effect of the lattice sums and the radial integrals (Fig. 3) produces an evolution of the c.f. effect similar to the observed one.

The energy of the excited configurations of opposite parity, e.g. $6 s$ and $5 d$, may also influence the c.f. parameter values. A decrease in the energy of these configurations should strengthen the c.f. effect by increasing the mixing of the opposite parity terms to the $4 f$ wave functions. The energy of e.g. the $4 f^{N-1} 5 d$ configuration has a discontinuation in the middle of the $\mathrm{RE}^{3+}$ series (Fig. 4 [20]) which may be one of the reasons of the evolution of the c.f. effect.

\section{Acknowledgments}

The authors are indebted to Dr. H. Mutka (ILL, Grenoble, France) for measuring the inelastic scattering spectra of the $\mathrm{Nd}^{3+}$ ion in NdOF, to Prof. W. Stręk and Dr. P. Deren (Polish Academy of Sciences, W. Trzebiatowski Institute of Low Temperature and Structure Research, Wroclaw, Poland) for measuring some of the luminescence spectra and to Prof. Jussi Valkonen (University of Jyväskylä) for the use of the XPD equipment. Financial aid from the Academy of Finland (project \#4966) to the authors (J.H. and E.S.) is gratefully acknowledged. Part of the work has been carried out within the Graduate School of Materials Science.

\section{References}

[1] W.T. Carnall, G.L. Goodman, K. Rajnak, R.S. Rana, J. Chem. Phys. 90, 3443 (1989).

[2] J. Hölsä, L. Niinistö, Thermochim. Acta 37, 155 (1980).

[3] J. Hölsä, E. Sällynoja, K. Koski, H. Rahiala, J. Valkonen, Powder Diffr. 11, 129 (1996).

[4] O. Antson, L. Beaury, J. Derouet, J. Hölsä, E. Säilynoja, H. Mutka, P. Porcher, submitted to Inorg. Chem..

[5] J. Hölsä, E. Kestilä, J. Chem. Soc., Faraday Trans. 91, 1503 (1995).

[6] J. Hölsä, E. Kestilä, R. Saez-Puche, P. Dereń, W. Stręk, P. Porcher, J. Phys, Condens. Matter 8, 1575 (1996).

[7] R.A. Young, The Rietveld Method, Oxford University Press, New York 1993.

[8] A. Sakthivel, R.A. Young, Program DBWS-9006PC for Rietveld Analysis of X-ray and Neutron Powder Diffraction Patterns, Georgia Institute of Technology, Atlanta 1991.

[9] B.G. Wybourne, Spectroscopic Properties of Rare Earths, Interscience, New York 1965.

[10] H. Crosswhite, H.M. Crosswhite, B. Judd, Phys. Rev. 169, 130 (1968).

[11] J.L. Prather, Monograph 19, US National Bureau of Standards, Washington 1961.

[12] K. Lesniak, J. Phys., Condens. Matter 2, 5563 (1990).

[13] R.P. Leavitt, C.A. Morrison, D.E. Wortman, Report TR-1679, Harry Diamond Laboratories, Adelphi (USA) 1975.

[14] A.J. Freeman, J.P. Desclaux, J. Magn. Magn. Mater. 12, 11 (1979).

[15] N.C. Chang, J.B. Gruber, R.P. Leavitt, C.A. Morrison, J. Chem. Phys. 76, 3877 (1982). 
[16] D.H. Metcalf, T.A. Hopkins, F.S. Richardson, Inorg. Chem. 34, 4868 (1995).

[17] Y.Y. Yeung, D.J. Newman, J. Chem. Phys. 86, 6717 (1987).

[18] M.F. Reid, J. Chem. Phys. 87, 2875 (1987).

[19] E. Rukmini, C.K. Jayasankar, M. Reid, J. Phys., Condens. Matter 6, 5919 (1994).

[20] L. Brewer, J. Opt. Soc. Am. 61, 1666 (1971). 